\section{Author`s Reply}

To the Editor,

We thank the authors for their valuable evaluation of our article entitled "Relationship between the presence of left atrial thrombus in patients with mitral stenosis and platelet-to-lymphocyte ratio" published in Anatol J Cardiol 2016; 16: 673-7 (1).

The aim of our study was to examine the relationship between the presence of left atrial (LA) thrombus and platelet-to-lymphocyte ratio (PLR), a marker of inflammatory process, in patients with rheumatic mitral valve stenosis (RMVS). This prospective cross-sectional study included patients with a mitral valve area less than $2 \mathrm{~cm}^{2}$. Patients were divided into two groups: those with and without LA thrombus. Because of the pathophysiological properties of the disease, it is normal for variables, such as atrial fibrillation (AF), mitral valve area, mean gradient, and left atrial volume, to be different between the groups.

The relationship between $\mathrm{AF}$ and LA thrombus formation is also strong in patients with RMVS. It is known that rheumatic heart diseases are autoimmune inflammatory pathologies and that inflammation subclinically continues $(2,3)$. One of the primary aims of the study was to examine the relationship between thrombus formation and PLR, which is a marker of inflammatory activity. While the presence of AF was associated with LA thrombus formation, this was independent of PLR levels. While its role of inflammation in the pathophysiology of $A F$ is known, the presence of paroxysmal AF, ambulatory ECG recordings, or time of AF were not recorded as they were not the primary aim of the study. Even though the relationship between inflammation and thrombus formation does not mean that there should be a relationship between PLR and AF, the presence of a link between $\mathrm{AF}$ and PLR may have been affected by the lack of these prelated parameters. At the same time, regression analysis results demonstrated that the relationship between PLR levels and AF continued to be independent of variables, such as $\mathrm{AF}$, mitral valve area, mean gradient, and left atrial volume.

\section{Erdal Belen}

Department of Cardiology, Okmeydanı Training and Research Hospital; İstanbul-Turkey

\section{Reference}

1. Belen E, Özal E, Püsüroğlu H. Relationship between the presence of left atrial thrombus in patients with mitral stenosis and plateletto-lymphocyte ratio. Anatol J Cardiol 2016; 16: 673-7.

2. Gölbaşı Z, Uçar 0, Keleş T, Şahin A, Çağı K, Çamsarı A, et al. Increased levels of high sensitive $\mathrm{C}$-reactive protein in patients with chronic rheumatic valve disease: evidence of ongoing inflammation. Eur J Heart Fail 2002; 4: 593-5.

3. Chiu-Braga YY, Hayashi SY, Schafranski M, Messias-Reason IJ. Further evidence of inflammation in chronic rheumatic valve disease (CRVD): high levels of advanced oxidation protein products (AOPP) and high sensitive $\mathrm{C}$-reactive protein (hs-CRP). Int J Cardiol 2006; 109: 275-6.

\footnotetext{
Address for Correspondence: Dr. Erdal Belen Okmeydanı Eğitim ve Araştırma Hastanesi Kardiyoloji Bölümü, Darülaceze Cad. No: 2534384 Okmeydanı, Şişli, İstanbul-Türkiye

E-mail: belenerdal@gmail.com
} 